\section{Educación de calidad o detrimento de la universidad pública: construcción de una mirada crítica alrededor de las políticas públicas de educación superior de la década de los noventa ${ }^{1}$}

\author{
Diana Patricia Ortiz Castro ${ }^{2}$ \\ dianaportiz@hotmail.com \\ Universidad Distrital Francisco José de Caldas \\ Bogotá - Colombia \\ Artículo recibido: 28/04/2014 \\ Artículo aprobado: 20/06/2014
}

Para citar este artículo: Ortiz, P. (2014). Educación de calidad o detrimento de la universidad pública: construcción de una mirada crítica alrededor de las políticas públicas de educación superior de la década de los noventa.

Ciudad Paz-Ando, 7(1), 178-196

DOI: http://dx.doi.org/10.14483/udistrital.jour. cpaz.2014.1.a10

1 En este artículo se reflejan parte de los resultados obtenidos en la investigación titulada: Políticas de Acreditación en el Marco de la Universidad Distrital Francisco José de Caldas. Un Análisis Crítico (2007-2012).

2 Licenciada en Educación Básica con Énfasis en Ciencias Sociales e integrante del semillero de investigación CEATO TAKI ONGOY de la Universidad Distrital Francisco José de

\section{Quality education or detriment of the public university: Building a critical look around the 1990s public higher education policy}

\section{Educação de qualidade ou} detrimento da universidade pública: construindo um olhar crítico em torno de políticas públicas de ensino superior dos anos noventa.

\section{Resumen}

El presente artículo plantea un análisis respecto a las políticas públicas de educación superior de la década de los noventa que han venido transformado sustancialmente las relaciones entre la Universidad Pública y el Estado. De este modo, desde una visión crítica del paradigma modernizador, se presentan las principales transformaciones a las que han sido sometidas las Universidades mediante efectivos mecanismos de evaluación, supeditados a las políticas económicas dominantes. Conforme a lo planteado, al interior de las Universidades Públicas se vienen instalando una serie de reformas profundas que introducen cambios en los currículos, en las formas de financiación, en la orientación de las carreras, de la investigación y de la organización alterando el sentido y la finalidad de la Universidad Pública.

Palabras clave: Políticas Públicas, neoliberalismo, calidad, evaluación, privatización, educación superior. 


\begin{abstract}
This paper presents an analysis regarding higher education public policies along the 1990's, which have changed the relationships between public university and the State. Thus, from a critical perspective of the modernizing paradigm, this paper explains the main changes public universities had faced by effective evaluation means which depend on the predominant economic policies. According to the previous ideas, within public universities, a set of deep reforms have been installed, which introduce changes into curriculums, financing methods, programs' orientation, research, and organization. Those reforms modify the sense and purpose of public university.
\end{abstract}

Keywords: Public policies, neoliberalism, quality, evaluation, privatization, higher education.

\section{Introducción}

L a investigación (Ortiz, 2014) cuyos resultados se da cuenta en el presente artículo fue trazada con la finalidad de develar el papel que vienen cumpliendo las políticas públicas en la consolidación de un modelo educativo neoliberal. La investigación pretendía identificar las principales tendencias en la política pública educativa para de esta manera, determinar los alcances y repercusiones que éstas han tenido para el caso específico de la Universidad Distrital Francisco José De Caldas (FJDC).

En lo que concierne al presente artículo, resulta necesario mostrar las líneas generales que direccionan estas políticas y los organismos promotores de este tipo de reformas ma-

\section{Resumo}

Este artigo apresenta uma análise das políticas públicas em matéria de educação superior na década dos anos noventa, que transformou substancialmente a relação entre a Universidade Pública e o Estado. Assim, a partir de uma visão crítica do paradigma da modernização, o texto apresenta as principais transformações a que são submetidas as universidades públicas, que dependem de avaliações efetivas das políticas económicas dominantes. Conforme o colocado, dentro das universidades públicas se vêm instalando uma série de reformas profundas que introduzem mudanças nos currículos, nas formas de financiamento, na orientação profissional, na pesquisa, na forma de administração. Estas reformas alteram o sentido e propósito da Universidade Pública.

Palavras-chave: Políticas Públicas, neoliberalismo, avaliação da qualidade, privatização, ensino superior.

terializadas a nivel mundial. La tesis a desarrollar define la evaluación y la calidad educativa como los pilares sobre los cuales el neoliberalismo irrumpe en las políticas institucionales universitarias, siendo así los instrumentos con los cuales el Estado relega en otras instancias su rol en torno a la educación superior, "mientras que las fuerzas del mercado se fortalecen y definen aspectos importantes de la organización y oferta del sistema de educación superior" (Guido, 2005, p.1). Al respecto conviene decir que las fuertes presiones económicas ejercidas mediante la evaluación sobre el sistema de educación superior nacional han conllevado a la pérdida del ethos y del sentido que caracterizaban la universidad pública. 


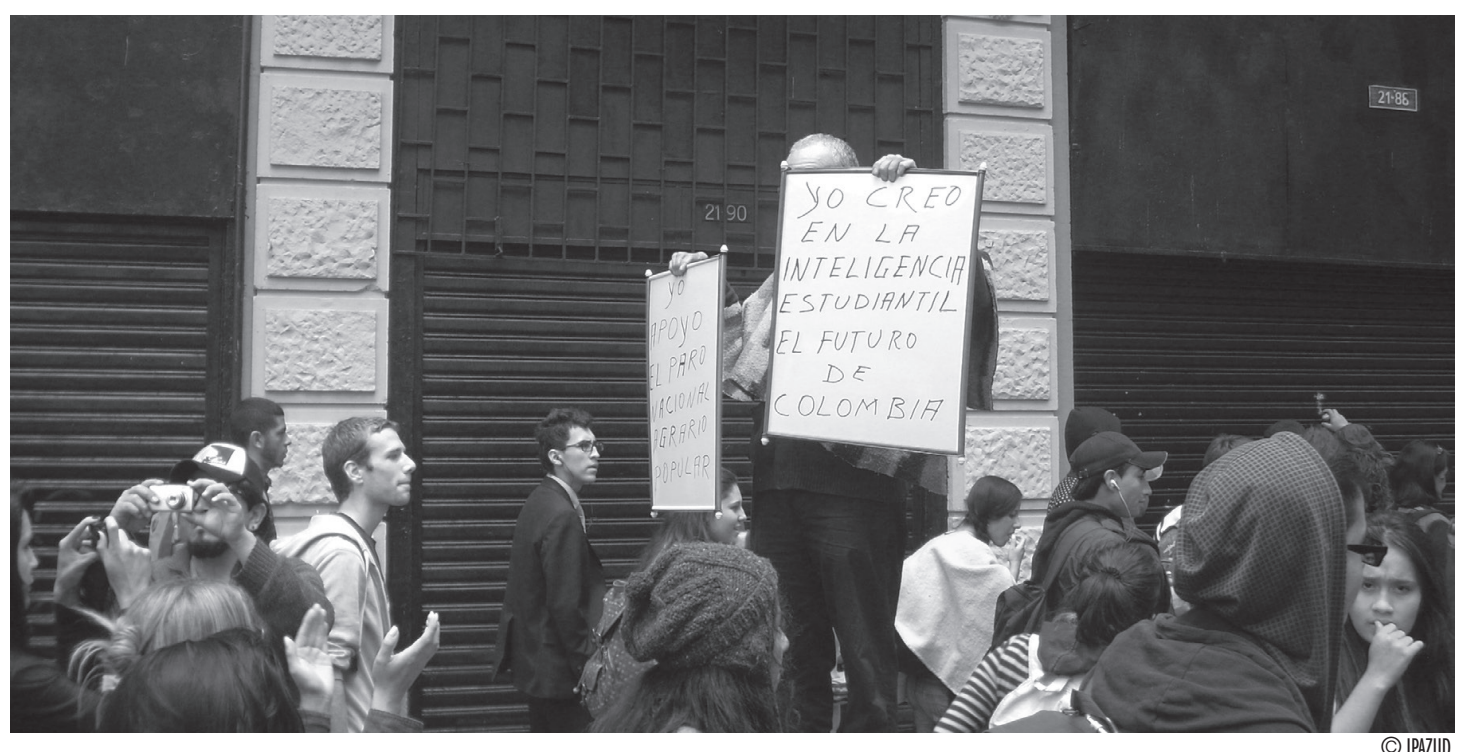

Ante esta crisis de sentido, la influencia de los proyectos de carácter internacional, ajenos a las necesidades del país, han impuesto el paradigma modernizador y de mercado, provocando así, una visible contradicción entre la autonomía (la cual queda reducida a lo administrativo) y las exigencias de privatización, competitividad, eficiencia y capitalización.

El documento consta de cuatro apartados. En el primero se realiza una aproximación histórica al neoliberalismo. En el segundo se delimitan las exigencias construidas por el Banco Mundial (BM), el Fondo Monetario Internacional (FMI), el Banco Interamericano de Desarrollo (BID) y la UNESCO para el sector de la educación superior; con el propósito de evidenciar la manera como estos arsenales conceptuales, teóricos y metodológicos han determinado la explosión de reformas y transformaciones de las universidades, en la década de los noventa. Dentro de este apartado se construye una reflexión frente a la manera como estas instituciones financieras han favorecido los proyectos encaminados a modificar las estructuras tradicionales de financiamiento hacia la reducción de los recursos públicos provenientes del Estado, fortaleciendo así la intervención privada. En el tercer apartado se analiza el concepto de calidad educativa, con el propósito de develar el tipo de educación subrepticio incorporado en esta categoría. Finalmente, se establecen una serie de reflexiones frente a los retos que asume la universidad pública actualmente, como respuesta a las exigencias de carácter internacional que le imponen convertirse en una institución especializada en la venta de servicios, lo cual hace que la diferenciación entre universidad pública y universidad privada sea cada vez más tenue.

\section{Antecedente histórico: emergencia del neoliberalismo}

El neoliberalismo ha sido el concepto acuñado para delimitar una corriente económica y política que tuvo lugar tras la Segunda Guerra Mundial, y cuyos presupuestos encarnan una fehaciente oposición hacia las políticas emanadas del Estado de Bienestar y más 
propiamente hacia el intervencionismo estatal. Por tal razón, en el marco de la educación superior se ha privilegiado paulatinamente el traspaso de las obligaciones del Estado hacia otras instancias, en su mayoría privadas, como los sectores productivos. En otras palabras, se han hecho todos los esfuerzos posibles para disminuir la capacidad del Estado de coordinar estratégicamente el desarrollo de la educación superior.

Ahora bien, es preciso recordar que el Estado de Bienestar para finales de los años 40 constituyó una respuesta a la crisis ulterior a la Segunda Guerra Mundial, por medio de la cual se pretendía generar estabilidad mercantil mediante la aplicación de políticas fiscales y monetarias con las cuales el Estado pudiese intervenir de forma deliberada en la regulación de los ciclos económicos.

Si bien las políticas fiscales y monetarias keynesianas lograron consolidar una estabilidad en los precios a través de mecanismos de control de capitales, a finales de la década de 1960 estas medidas empiezan a entrar en crisis: "el crecimiento tanto del desempeño como de la inflación se disparó por doquier anunciando la entrada en una fase de estanflación global que se prolongó durante la mayor parte de la década de 1970" (Harvey, 2007, p.18).

Conforme a lo expuesto, y contando con los imperativos emanados de los acuerdos de Brettow Woods: el librecambismo, el establecimiento del dólar como moneda de reserva global, el surgimiento del FMl y del BM, resultaba notoria la instalación hacia el año de 1970 de una nueva tendencia económica conocida como neoliberalismo. En consecuencia, el neoliberalismo pasa a acomodarse de manera progresiva en el panorama mundial; en unas ocasiones, los Estados acogieron estas políticas de manera voluntaria, y en otras, obede- ciendo a fuertes presiones externas ejercidas por estos organismos, de tal manera que se han visto obligados a ajustar sus prácticas a tales premisas (Harvey, 2007, p.10).

Por consiguiente, el neoliberalismo ha sido considerado un proyecto económico que ha viabilizado el afianzamiento y la restauración del poder de ciertas elites económicas de los países desarrollados (quienes no han escatimado ningún esfuerzo para lograrlo) ${ }^{3}$, esto a través del fortalecimiento de las libertades correspondientes a las multinacionales, a las empresas e indiscutiblemente al capital financiero. Los grandes defensores del neoliberalismo se han caracterizado prioritariamente por la privatización de activos de forma sistemática.

El aparataje desplegado para restaurar el poder de clase durante la década de 1980, proceso consolidado solo hasta 1990, se desarrolló a través de las siguientes trasformaciones: la primera, el giro a la financiarización, el cual comenzó en 1970 con el aumento de la inversión extranjera directa y la expansión e innovación de los mercados financieros como medios para la acumulación de capitales como sus principales medidas; y la segunda, la creciente movilidad geográfica del capital. En esta etapa, el papel desempeñado por el BM y el FMl fue muy importante, ya que estos tomaron el grado de neoliberalización de los países como indicador para medir la calidad del clima de los negocios. La presión por implementar las políticas neoliberales fue bastante agresiva, sobre todo en los países del tercer mundo. Finalmente, la difusión del monetarismo fue el condicionante ideológico de mayor importancia, teniendo en cuenta que el énfasis en las universidades estadounidenses estaba puesto

3 En Estados Unidos e Inglaterra el neoliberalismo propició la restitución del poder de las clases dominantes a diferencia de otros países en donde se crearon las condiciones para la formación de una clase capitalista apoyada en la organización empresarial. 
en formar economistas de numerosos países en esta doctrina (Harvey, 2007).

En consecuencia, las primeras incidencias del neoliberalismo se manifestaron en las políticas públicas de Estados Unidos e Inglaterra en donde el énfasis en el individualismo, la protección de la propiedad privada y las políticas de desregulación de todas las áreas son algunos de sus elementos más característicos, todo lo cual se fue convirtiendo en el armazón que permitiría a las elites mercantiles la consolidación del poder monopolista (Harvey, 2007).

Considerando lo anterior, el BM acude a la educación superior con el propósito de promover la competitividad para obtener una fuerza laboral bien instruida en coherencia con el sistema económico descrito. En efecto, se piensa que el desarrollo económico depende, en gran medida, de la adaptación del sistema de educación superior a las necesidades de los sectores productivos. Por tal razón, esta institución financiera asume los postulados de la teoría del capital humano, desde la cual la educación debe ser vista como una inversión en los recursos humanos de los países en desarrollo ${ }^{4}$. El énfasis está puesto en acomodar los cuadros productivos en las instituciones universitarias con el fin de ajustar los currículos a las necesidades de estos sectores (Banco Mundial, 1995). Concretamente, "se pide al sistema de enseñanza superior que sea flexible y responda a los imperativos del mercado del trabajo" (Sanz, 2006, p.64).

De acuerdo a lo anterior, las políticas laborales en auge han promovido la flexibilización como principal instrumento de desregulación

$4 \quad$ La teoría del capital humano fue formulada y desarrollada en la década de los setenta por Teodore W. Schultz y Gary S. Becker (premios nobel de economía en 1979) Según esta teoría: "después del nivel básico, la demanda de la educación está determinada por dos variables: los costos individuales directos e indirectos de adquirirla, y las variaciones que esta educación producirá posteriormente sobre las oportunidades de empleo y los niveles de ingreso de quien la adquiere" (Maldonado, 2005, p.68). del mercado laboral. El gran ejército del trabajo que se encuentra a disposición de las empresas resulta un condicionante provechoso para el control de la fuerza de trabajo así como de la elevada tasa de explotación laboral a manos de las elites neoliberales. Los contratos a corto plazo que sostienen los mercados laborales flexibles responden a esta lógica. En suma, se vislumbra cómo las políticas públicas fueron pareciéndose cada vez más a las políticas empresariales.

\section{Constitución de un modelo educativo neoliberal en las políticas públicas de educación superior}

En la misma línea de Volcker y Reagan en Estados Unidos (1981-1989), y de Margaret Thatcher en Inglaterra (1979-1990), en la mayoría de países de América Latina se ha establecido un marco de políticas educativas coherentes con las necesidades de la neoliberalización. En tal sentido, el BM ha provocado el aumento de la oferta privada y el desarrollo de instituciones no universitarias (institutos politécnicos, institutos profesionales y técnicos de ciclos cortos) bajo la política de la individualización de los $\operatorname{costos}^{5}$, teniendo en cuenta el aumento de la tasa de matrícula de la década de 1990. Según el BM, estas instituciones "responden en forma flexible a las demandas del mercado laboral y están vinculadas a los programas universitarios mediante mecanismos apropiados de transferencia, como los sistemas de créditos" (1995, p.6). Con esta estrategia se ha pretendido optimizar los recursos públicos y afianzar la gerencia

5 "La mayor participación de los estudiantes en el financiamiento de sus estudios, un generalizado recorte en algunos gastos que hasta hacia unas décadas asumían las universidades públicas (de alimentación y de vivienda), el surgimiento de sistemas de crédito educativo y la promoción de becas son las consecuencias más visibles de esta política" (Banco Mundial, 1995, p.8). 


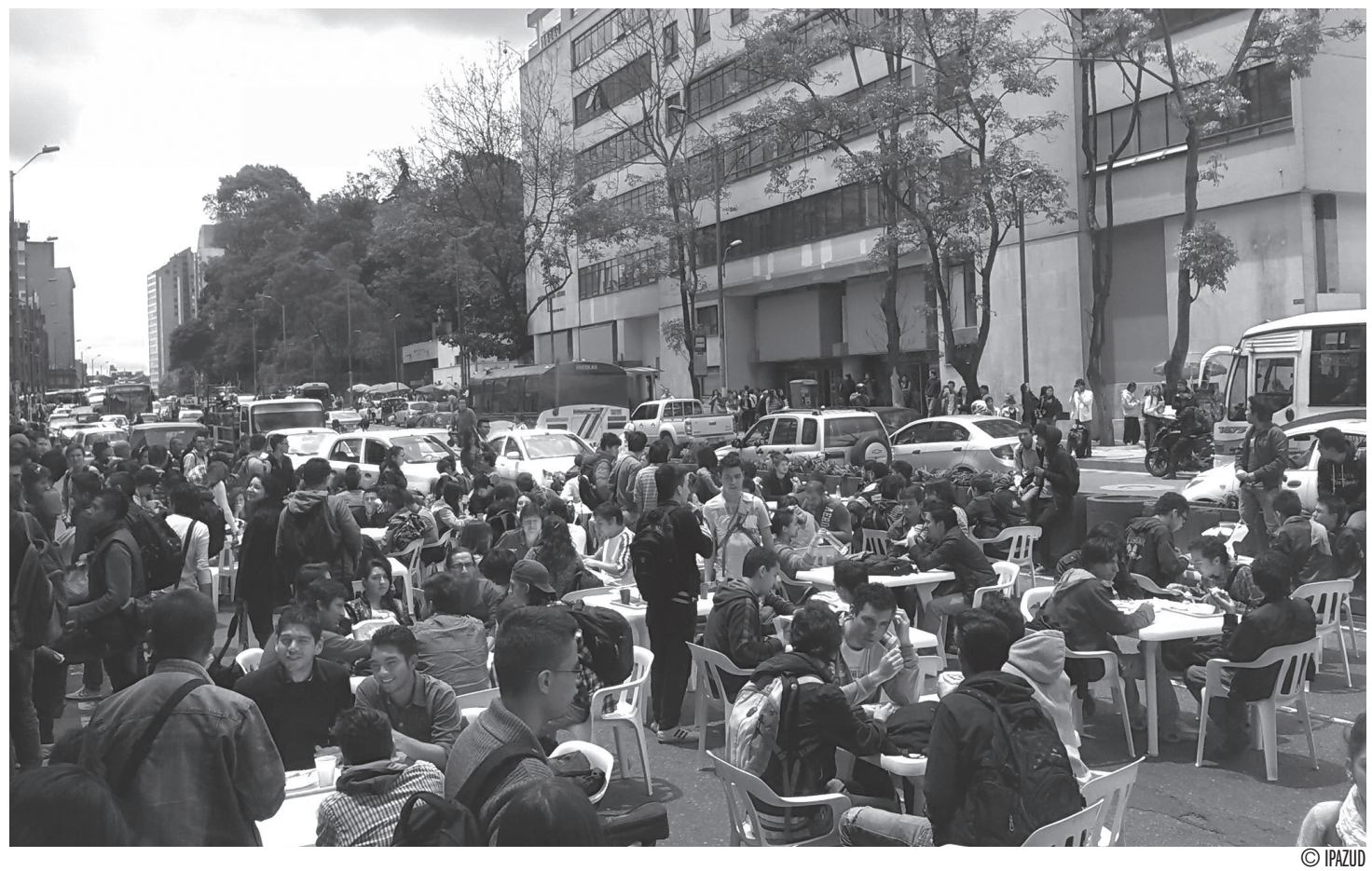

de los proveedores privados, transformando sustancialmente el sentido y la finalidad de la educación. Desde este enfoque, la educación superior se ha convertido en una mercancía sujeta a las determinaciones de la demanda.

Ahora bien, la estrategia de fomento de la oferta privada de la educación superior es considerada como la más acorde con la política del Estado desertor, promovida por la economía neoliberal. En efecto, el BM plantea que,

los gobiernos pueden fomentar el desarrollo de la educación terciaria privada a fin de complementar las instituciones estatales como medio de controlar los costos del aumento de la matrícula de la educación superior, incrementar la diversidad de los programas de enseñanza y ampliar la participación social en el nivel terciario. $(1995$, p.6)

Igualmente, sostiene la necesidad de acompañar estas medidas con mecanismos de acreditación, fiscalización y evaluación que permitan vigilar el uso adecuado de los recursos para el caso de las instituciones públicas y controlar la explosión de instituciones privadas. De hecho, a través del análisis de lo propuesto por este organismo, se puede inferir su preocupación por relegar el papel del Estado, de allí que sus principales recomendaciones estén dirigidas a la eliminación de aquellos subsidios que no estén relacionados con la instrucción, el cobro y venta de servicios, la diversificación de fuentes de financiamiento y la implementación de métodos de fiscalización que permitan subsanar el vacío que ha dejado el desmantelamiento del Estado.

De acuerdo a lo anterior, el Consenso de Washington ${ }^{6}$, redactado hacia 1990, promo-

6 El consenso de Washington, que en realidad son un conjunto de acuerdos elaborados por los decisores de las políticas financieras en Estados Unidos y en donde no se tienen en cuenta a los países en vías de desarrollo, quienes reciben sus efectos, es el mejor ejemplo de la restauración del poder económico que tenía en mente la minoritaria elite económica consolidada en Estados Unidos. 
vía la política de reducción sistemática de la intervención del Estado en los asuntos monetarios, en consonancia con las políticas de Estado implementadas por Margareth Thatcher y Ronald Reagan; la liberalización de los flujos financieros, así como la expansión geográfica de los capitales por medio de la creación de la Organización Mundial del Comercio (OMC) en 1995, se mostraban como las recomendaciones de orden mundial más pertinentes considerando la implosión del sistema socialista.

A partir de los acuerdos efectuados por la $\mathrm{OMC}$ en torno a la liberalización del comercio de servicios, se empieza a recurrir al mercado como proveedor de la educación superior "en un mercado formal de orden internacional y determinados gobiernos ya han suscrito compromisos de liberalización en materia de servicios educativos o están invitando a las distintas empresas de formación a que se instalen en sus países" (Sanz, 2006, p.64).

Considerando lo expuesto, el modelo neoliberal en las políticas públicas de educación superior tuvo gran acogida durante la década de los ochenta y los noventa, pues es en este periodo donde se vivencia "el paso de una estructura en expansión a una de contracción" (Didriksson, 2000, p.60). La primera promovió la instalación de un gran número de Instituciones de Educación Superior (IES) en todo el mundo, y el crecimiento desaforado de las mismas sin la infraestructura y los recursos necesarios provocó la propagación de un sistema de educación superior con profundas rupturas y desigualdades. Ante esa situación, las políticas públicas educativas inscribieron los sistemas de evaluación como herramientas para controlar o contraer esa explosión de instituciones y materializar el modelo privatizador construido por los países desarrollados.

La segunda estructura, conocida como de contracción, emerge como respuesta al déficit en el erario público provocado por el establecimiento de la doctrina neoliberal en todas las instancias de la sociedad. En consecuencia, los recortes presupuestales para el detrimento y pauperización de lo público promovidos por estas políticas neoconservadoras, llevaron a las universidades públicas a dirigir sus proyectos hacia otros sectores como las empresas, con lo cual la organización interna y las lógicas propias de estas instituciones se vieron comprometidas. En términos del investigador Axel Didriksson,

en esta fase la universidad ve alterada su orientación, ante lo cual ocurren profundos cambios internos en las formas de gobierno, en la comprensión de la autonomía académica y en la calidad y mecanismos de trasferencia de los conocimientos y la tecnología hacia la sociedad. La orientación básica es hacia el mercado, y la universidad pública se convierte en una institución de servicios. (2000, p.60)

En consonancia con esas vicisitudes, la presidencia de Reagan, de acuerdo al FMI, otorgó interés a la disposición de privilegiar a las entidades financieras por sobre todos los aspectos del gobierno, "la creación de un <<clima óptimo para los negocios >> (...), significó utilizar los recursos públicos para construir infraestructuras adecuadas (...) que fueron acompañadas de incentivos fiscales y de subvenciones destinadas a las empresas capitalistas" (Harvey, 2007, p.53). Esto comprobó la nueva funcionalidad del Estado, la de velar por la libertad de las empresas y el buen funcionamiento del sistema financiero aún en detrimento de los derechos colectivos y de la población. Con todo esto, se puede inferir que las medidas adoptadas en Estados Unidos durante el gobierno de Ronald Reagan y George Bush fueron 
asimiladas en todo el mundo. Conceptos como calidad, mediocridad, excelencia, educación e investigación para el desarrollo económico se encuentran hoy en el centro del debate de la educación superior.

Atendiendo las prioridades estipuladas por la economía imperante, el FMI construyó las siguientes recomendaciones para los sistemas de educación superior:

- Financiar la educación superior mediante impuestos en una pequeña escala (de, digamos, el $1 \%$ del PIB) con el fin de proporcionar educación superior de buena calidad a algunos estudiantes o de menor calidad a más estudiantes.

- Basarse en el financiamiento privado, aceptando que ello limitaría el acceso a los estudiantes cuyas familias puedan asumir los pagos y, acaso, a un cierto número de estudiantes becados.

- Introducir un régimen de préstamos a pequeña escala, aceptando que a este le corresponderá por defecto una tasa de interés alta y unos costos administrativos elevados.

- Emplear los recursos procedentes del contribuyente para sufragar dos años de educación universitaria y dejar el resto al financiamiento privado.

- Servirse de la asistencia al desarrollo para lograr el equilibrio entre los métodos mencionados. (Barr, 2005, p.37)

Podría decirse que la creciente expansión cuantitativa de las IES en la década de los noventa, la diversificación del financiamiento de las mismas como respuesta al recorte público, la creciente privatización, la vinculación de la universidad pública con el sector productivo, específicamente con la empresa, el cambio en la gestión universitaria, las nuevas re- laciones entre educación público-privada, los métodos de fiscalización (para la optimización de los recursos públicos con procedimientos de eficacia y eficiencia) y la combinación entre educación formal, informal y a distancia, son algunas de las consecuencias de la intromisión del modelo neoliberal en la educación, manifiestas en las políticas educativas de numerosos países.

Habría que tener en cuenta que en América Latina y el Caribe este conjunto de cambios se configuraron históricamente a través del paso del modelo cepalino de sustitución de importaciones y fortalecimiento del Estado a las sucesivas dictaduras que sirvieron de instrumento para la implementación de las reformas neoliberales (López, 2002). A diferencia de los países europeos y de Estados Unidos, en donde la serie de reformas se implementaron en el interior de las universidades a manera de innovación, en América Latina han sido el resultado de políticas asumidas desde afuera, lo que obviamente ha implicado serias dificultades. De acuerdo con estos argumentos, para la educación superior en América Latina se han priorizado reformas en torno a la organización del trabajo, a la preparación de una mano de obra más calificada para la competencia global, a la generación de calidad y a la formación en nuevos campos como el manejo de las tecnologías de la información, la implementación de mecanismos de rendición de cuentas y nuevas formas de enseñanza como la virtualización para la optimización de costos. En palabras de López:

El modelo neoliberal en lo que a las universidades se refiere, implica lo siguiente: La universidad contrata con el Estado, con las empresas y con los padres de familia que tienen capacidad de pagar la educación de sus hijos. Todos exigen "niveles de excelencia", en conocimientos 


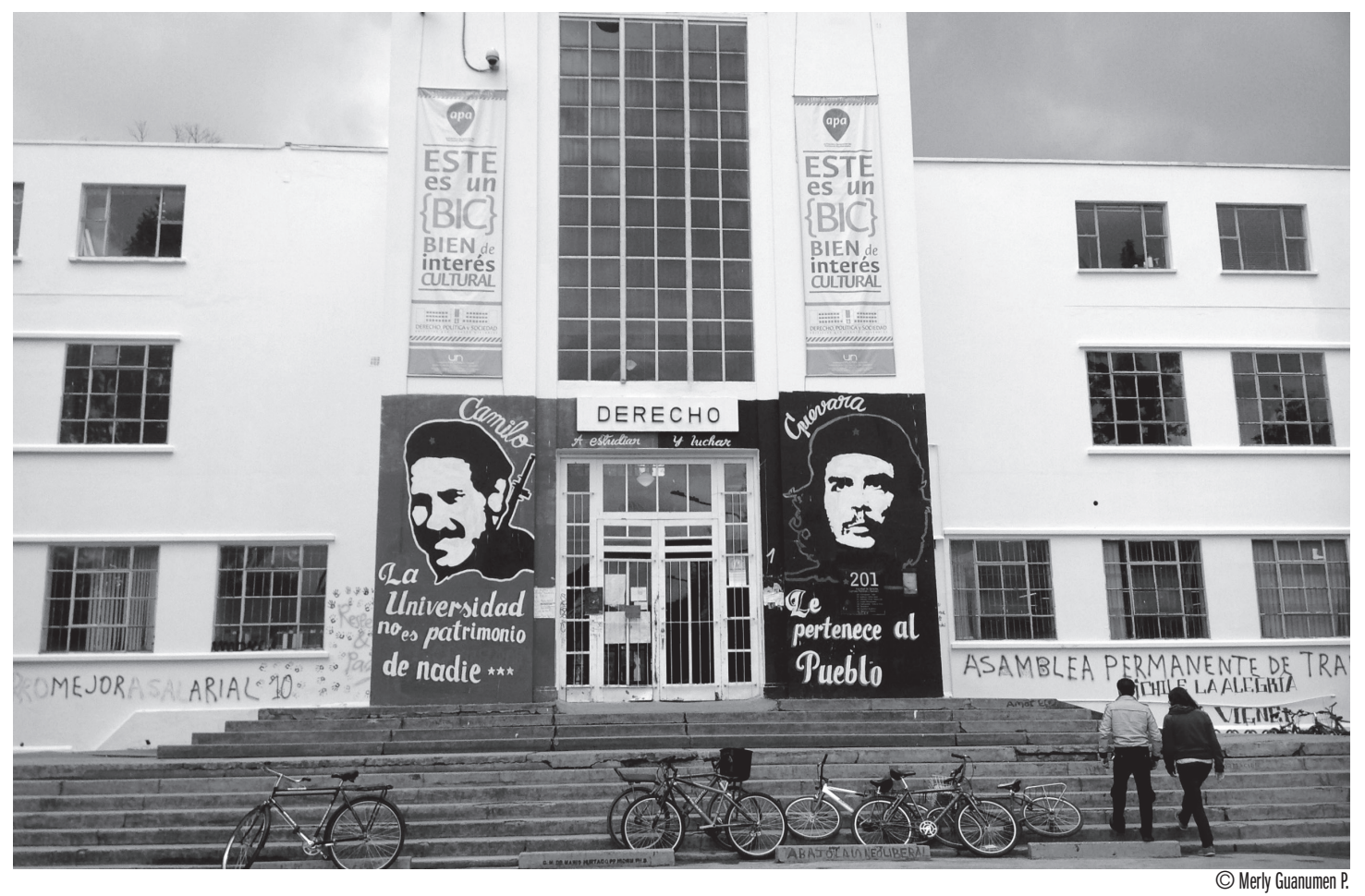

y saberes útiles a los mercados, y procuran no rebasar la demanda con sobreofertas de egresados que abatirían los sueldos y empleos. Esta nueva universidad neoliberal tiene una política llamada "humanitaria", mediante la cual fundaciones públicas o privadas ofrecen becas 0 créditos a los estudiantes pobres que no pueden costearse sus estudios. (...) Se piensa en términos de mercado y esto conduce a la creación de las universidades corporativas, en función de las necesidades de las corporaciones, como fue el caso pionero de la General Motors. (2002, p.19)

Además, según los estudios de las tasas de rentabilidad ${ }^{7}$ realizadas por el BM, las inversiones en la enseñanza básica y secundaria traen mayores beneficios que las inversiones realizadas en el nivel terciario; por tal razón, los préstamos otorgados conceden mayor atención a la enseñanza básica y secundaria que a la superior. Estos préstamos se encuentran en función de:

Apoyar los esfuerzos de los países para adoptar reformas de política que permitan al subsector funcionar en forma más eficiente y a un menor costo público. Se seguirá dando prioridad a los países dispuestos a adoptar un marco de políticas en materia de educación superior que haga hincapié en la estructura diferenciada de las instituciones y una base de recursos diversificada y mayor importancia a los proveedores y al financiamiento privado. En estos países, los préstamos del Banco destinados a la enseñanza terciaria apoyan reformas de políticas sectoriales, desarrollo institucional y mejoramiento de la calidad. (Banco Mundial, 1995, p.14) 
Todas estas trasformaciones en las políticas sociales que parecieran sintomáticas conllevan a grandes reformas estructurales entre las que vale la pena mencionar la dependencia creciente de los consorcios público-privados, una de las medidas más implementadas por Margaret Thatcher en Inglaterra ${ }^{8}$, y que aprueba la gerencia de empresarios y corporaciones en actividades propias del Estado como la redacción de legislaciones, la creación de políticas públicas y el establecimiento de marcos normativos.

Para el caso de los países en desarrollo, los Estados neoliberales han presentado desde sus inicios políticas inestables y contradictorias que en numerosos casos se han distanciado de los planteamientos teóricos de este modelo (Harvey, 2007). En tal sentido, como ha sido recomendado por los organismos multilaterales, el Estado ha intervenido a favor del mercado en el contexto de una economía en la que se supone no debe ser intervencionista. Es así como el Estado pasa de ejercer control directo a crear el ambiente favorable al desarrollo financiero, por medio de la creación de políticas que permiten cubrir de forma eficiente las necesidades de investigación y enseñanza viables al proyecto de desarrollo económico. En términos del Banco Mundial,

se ha demostrado que el éxito de la ejecución de las reformas de la educación superior depende de lo siguiente: 1) el establecimiento de un marco coherente de políticas; 2) mayor apoyo en los incentivos y mecanismos orientados al mercado para aplicar las políticas, y 3) mayor autonomía administrativa de las instituciones públicas. (1995, p.10)

8 Por medio de la creación de instituciones cuasi gubernamentales se buscaba la inserción de intereses empresariales en el ejercicio del gobierno (Harvey, 2007).
Con estos imperativos se espera que las universidades públicas fijen derechos de matrícula, contraten y despidan personal, vendan servicios y oferten actividades que les permitan sobrellevar sus déficits económicos.

Estas formas políticas inestables pueden ser atribuidas conforme a lo anteriormente anunciado, a la tensión entre las dinámicas internas y las fuerzas externas que no son las mismas en todos los países. Aunque el neoliberalismo posee amplias universalizaciones que pretende aplicar en numerosos contextos, lo cierto es que las determinaciones de carácter social e histórico en cada país definen el papel del Estado en el marco de esta economía. En Chile, por ejemplo, fueron las clases altas las que solicitaron apoyo de Estados Unidos para dar paso al golpe militar, los tecnócratas de este país formados en Estados Unidos cumplieron muy bien su papel en la configuración de dicho modelo.

Términos como "neoconservadurismo", "neoautoritarismo", "extremismo mercantil" o "modernización nacional" son en consecuencia formas de poder muy relacionadas en cuanto a economía neoliberal se refiere. Modelos aplicados contradictoriamente ${ }^{9}$ ante la necesidad de arbitrar el caos de los intereses individuales y de salvaguardar los Estados neoliberales frente a los conflictos internos y externos que los amenazan. En tal vía, la militarización ha sido considerada como respuesta al caos y garantía de las libertades del mercado.

Por su parte, el BID al brindar recursos al desarrollo de la educación superior en países de América Latina y el Caribe, ha reproduci-

\footnotetext{
9 Recordemos que uno de los pilares más difundidos del neoliberalismo sostiene que en dicho modelo económico no caben las formas autoritarias de ejercicio del poder; históricamente el neoliberalismo se ha distanciado de la teoría adoptando el autoritarismo como principal mecanismo para la conservación de su infraestructura económica. Este distanciamiento ha sido considerado como una de las principales contradicciones de este modelo (Harvey, 2007)
} 
do los lineamientos del BM y del FMI. El caso chileno es el que más se aproxima al ideal del BID, ya que contempla el financiamiento conjunto entre los sectores público y privado en una proporción de 30\% a 70\% respectivamente, según el desempeño y los índices de la institución (por acreditación, evaluación, entre otros).

El aumento en la demanda de cupos dentro del "mercado"10 educativo, para los intereses del BID, ha sido muy bien controlado cuando el Estado ha restringido la oferta pública y ha solventado esos requerimientos con la oferta desde instituciones privadas (enfocadas en el mercado laboral). Además, dicho organismo financiero ve con buenos ojos el incremento en el cobro de aranceles o matriculas en universidades públicas, y la parcial y paulatina privatización de algunas de estas universidades, en especial las que no resultan lo suficientemente competitivas. En lo que respecta al liderazgo académico, formación para las profesiones, formación técnica y perfeccionamiento y educación general, como funciones básicas dentro del sistema planteado por el BID, se considera que el mercado es el mecanismo de regulación más eficiente. De hecho, como afirman De Moura Castro y Levy en su trabajo sobre las disposiciones de dicho organismo para la última década del siglo pasado,

La formación profesional debe, en general, ser impulsada por la demanda económica y no tanto por la social o la política. Después de todo, el propósito principal es responder a la demanda específica del mercado, no a la presión de los estudiantes que desean obtener diplomas de educación superior. (1997, pp.16-17)
La educación técnica está destinada para el grueso de la población, ya que satisface las necesidades básicas del mercado laboral a muy bajo costo; por ejemplo, en docencia. Dentro de este panorama se excluyen las "artes liberales" (humanidades), como son denominadas por este Banco, es decir, aquellas carreras que se encargan de lo abstracto y general, dejando de lado la posibilidad de engendrar una masa pensante y critica; únicamente se contempla la dimensión del trabajador y no del profesional.

Cuando habla de los problemas que afronta la educación superior en cuanto al acceso, el BID hace más énfasis en las deficiencias de los niveles de educación inferiores que a las políticas financieras en este campo. En un caso como el nuestro, es evidente que un estudiante egresado de una institución pública de educación media tiene menos posibilidades de ingresar a una universidad pública que un estudiante de clase media o alta al que le impartieron educación integral. Sin embargo, cabe resaltar que el tema del financiamiento estatal no tiene menor relevancia. Ni qué hablar de los costosísimos programas de postgrado.

Como se puede observar, "mientras la libertad personal e individual en el mercado se encuentra garantizada, cada individuo es responsable y debe responder por sus acciones y de su bienestar" (Harvey, 2007, p.73). En eso consiste la política de individualización de los costos, en trasladar responsabilidades a los estudiantes y sus familias. En términos más precisos, el financiamiento proveniente de los ex alumnos, la ayuda externa, los organismos crediticios, las donaciones de la industria privada, actividades que generen ingresos como cursos de corto plazo, investigaciones contratadas por la industria y servicios de consultoría, son las medidas que ejemplifican la ausencia del Estado y su pre- 
cariedad frente al fortalecimiento de los derechos fundamentales.

Por su parte, muchas de las propuestas de la UNESCO, contrariamente a lo que pregonaban sus precursores quienes afirmaban que esta institución se caracterizaba por partir de un enfoque social, se encuentran encaminadas en la misma línea de organismos como el BM y el BID. Basta con observar las siguientes recomendaciones promovidas por la institución para darse cuenta de ello:

- La adopción del paradigma de aprender a aprender.

- El traslado del acento, en la relación enseñanza-aprendizaje, a los procesos de aprendizaje.

- El nuevo rol de los docentes, ante el protagonismo de los discentes en la construcción del conocimiento significativo.

- La flexibilidad curricular y toda la moderna teoría curricular que se está aplicando en el rediseño de los planes de estudio.

- La promoción de una mayor flexibilidad en las estructuras académicas.

- El sistema de créditos.

- La estrecha interrelación entre las funciones básicas de la universidad (docencia, investigación, extensión y servicios).

- La redefinición de las competencias profesionales.

- La reingeniería institucional y la gestión estratégica como componente normal de la administración universitaria. (Implementación de mecanismos que garanticen la eficacia y la eficiencia del presupuesto público)

- Los procesos de vinculación de la sociedad y sus diferentes sectores (productivo, laboral, empresarial, etc). (Tunnermann \& De Souza, 2003, p.19)
En síntesis, "el discurso de la gestión empresarial, discurso que pretende ser a la vez formal e histórico, global y situado, constituye hoy la forma por excelencia en la que el neoliberalismo se materializa y se comparte" (Boltanski \& Chiapello, 2002, p.53).

Con todo ello, el Estado en el marco de una economía neoliberal ha creado sistemas de mercado educativo bajo el supuesto de que la desregulación y la promoción de la competencia lograrían acabar con los trámites burocráticos, incrementar la eficiencia y la productividad, mejorar la calidad de la educación superior y reducir los costes. Pese a todos esos beneficios que anunciaban los grandes promotores del neoliberalismo, lo cierto es que se han agudizado problemáticas como la desigualdad social y la pobreza ${ }^{11}$, sobre todo en países en vía de desarrollo. No se puede ignorar el hecho de que las profundas contradicciones generadas por este modelo han puesto en duda su viabilidad para el futuro de la educación superior. En efecto, actualmente asistimos al surgimiento de variadas formas de lucha social y política,

Cuanto más se reconoce al neoliberalismo como una fallida retorica utópica que enmascara un exitoso proyecto para la restauración del poder de la clase dominante (en beneficio de los países desarrollados), más se tienden los cimientos de una resurgencia de movimientos de masas expresando demandas políticas por la igualdad y aspirando a la justicia económica, el comercio justo y una mayor seguridad económica. (Harvey, 2007, p.210)

11 Según los estudios realizados por el BID "en los noventa se ha producido una extensión de la pobreza a más de 150 millones de latinoamericanos que equivale a cerca del $33 \%$ de la población que percibe menos de dos dólares diarios, mínimo necesario para cubrir las necesidades básicas de consumo. En 1998, pese a que el PIB creció en un $2.6 \%$, la desocupación en la región aumento de $7.2 \%$ a 8.4\%" (López, 2002, p.31). 


\section{La calidad del producto educativo: un sistema de medición de la educación superior como servicio}

Como ha sido expuesto, se han tomado como ejes de la acción estatal la evaluación de la educación superior, el mejoramiento de la calidad educativa a través del apoyo a proyectos, la apertura de las instituciones universitarias, la descentralización administrativa, la implementación del sistema de acreditación, y la articulación de las necesidades particulares al marco de la globalización y la competencia mercantil (Mendoza, 1997, p.74).

De acuerdo a lo anterior, es a partir de la Ley 30 de 1992 que se instalan estos procesos en Colombia. Básicamente, se emplean como el medio de ajuste y reestructuración de la educación superior, con lo cual se espera crear organismos independientes de fiscalización para formular y supervisar las políticas de educación superior, orientar las asignaciones presupuestarias y evaluar el desempeño de las universidades públicas en beneficio de los requerimientos económicos concertados en el modelo internacional de educación terciaria (Banco Mundial, 1995). Lo anterior ha suscitado una serie de disputas y contradicciones en las comunidades académicas, pues para algunos, el empleo de un sistema nacional de evaluación fortalece la calidad educativa, mientras que otros consideran que la evaluación sólo puede ser vista como un mecanismo de control empleado por el Estado en perjuicio de la universidad pública.

Desde esa línea argumentativa, Liliana del Basto cuestiona el modelo de universidad instaurado en los últimos años, planteando como argumento central la problemática pérdida del ethos universitario que se había construido históricamente, y que en el mar- cido a la rendición de cuentas. Por tal razón la autora sostiene que "el reflexionar sobre la universidad obliga a asumir, como imperativo, el recuperar el sentido de su espíritu original. Más que nunca se hace necesario volver la lista hacia atrás e indagar en torno al concepto y función de la universidad" (2004, p.6).

En ese contexto, el análisis de las políticas públicas en materia educativa resulta relevante, puesto que permite conocer el grado de apropiación y valoración con que amplios sectores de la sociedad materializan sus expectativas y requerimientos frente a la educación superior y, de igual forma, permiten entrever el grado de satisfacción de la población frente a las decisiones y alternativas ejecutadas por el Estado en esta materia. Para el caso colombiano, las políticas públicas en este sector han presentado un carácter difuso, debido, entre múltiples factores, a que en muchas ocasiones han sido tratadas de manera arbitraria por parte del gobierno de turno. A esto se suman varias problemáticas específicas del sistema político colombiano, tales como la inestabilidad del nivel administrativo, la carencia de presupuestos económicos, la influencia de los proyectos de carácter internacional que direccionan las políticas educativas y que no siempre corresponden a las necesidades de los escenarios locales de nuestro país, la crisis de representatividad y, de igual manera, la débil intervención y participación de la comunidad en la formulación de las mismas (Zamudio, 2008). Por tal razón, son insoslayables fenómenos como la exclusión y la inequidad que han impedido el afianzamiento de la ciencia y de la investigación en este país.

Ahora bien, es necesario remitirse al tipo de intereses que convergen en el concepto de calidad educativa como componente central del paradigma modernizador en la educación superior. Para empezar, se toman como refe- 


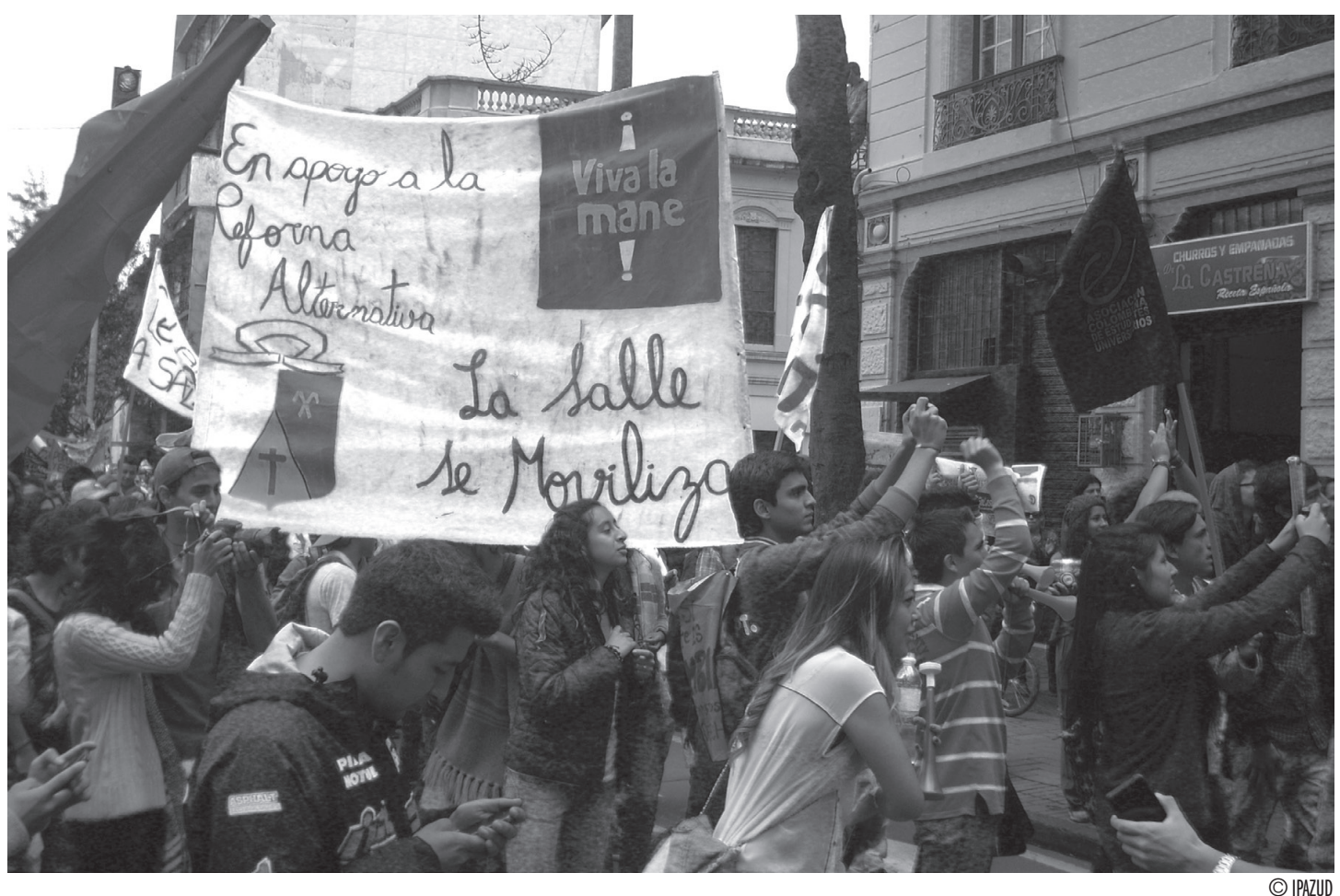

rentes internacionales el Modelo Europeo de Excelencia European Foundation For Quality Management (EFQM), y el Sistema de Gestión de Calidad propuesto por la Norma (ISO 9000) International Standarization Organization. A partir de estos modelos se observa el interés creciente por introducir los referentes conceptuales y metodológicos de la gestión empresarial en el funcionamiento de las universidades públicas. De modo que la calidad es definida como "la consecuencia de los insumos aplicados, de los procesos generados, del liderazgo, del desarrollo de personal y de los resultados, entre otros factores" (Sistema Nacional de Acreditación, 2005, p.23).

Según lo estipulado por el Sistema de Gestión ISO 9000, se deben emplear procedimientos como el marketing, de tal manera que se pueda incrementar la satisfacción del cliente, medir y monitorear el desempeño de las instituciones, e incrementar la eficacia y la eficiencia de su organización.
Conforme a estos referentes conceptuales, Boaventura de Sousa Santos identifica las tres crisis a las que se enfrentan las instituciones universitarias en la actualidad. La primera de ellas, la crisis de hegemonía, es definida como el resultado de la contradicción existente entre las funciones tradicionales de la universidad ${ }^{12}$ y las funciones que le son conferidas en el marco de la globalización económica. La segunda, la crisis de legitimidad producida por

El hecho de haber dejado de ser la universidad una institución consensual, frente a la contradicción entre la jerarquización de los saberes especializados de un lado, a través de las restricciones de acceso y certificación de las competencias, y de otro lado, por las exigencias sociales y políticas de la democratización de la

12 Entendidas como aquellas concernientes a la producción de los supremos valores del hombre, del pensamiento crítico y humanístico, de la formación de las elites, entre otras funciones. 
universidad y la reivindicación de la igualdad de oportunidades para los hijos de las clases populares. (2005, p.14)

Por último, la crisis institucional como resultado de la contradicción entre las exigencias de autonomía y los requerimientos de competitividad, eficiencia y capitalización que terminan ejecutando la mercantilización de la universidad, y afectando gravemente la universidad pública, teniendo en cuenta su dependencia al Estado.

De acuerdo con lo expuesto, De Sousa nos advierte sobre algunas de las consecuencias de las tres crisis mencionadas, resaltando la conversión de la universidad en un mercado y con ello la transnacionalización de los servicios universitarios, el paso de la educación como derecho a la educación como servicio, la reducción del financiamiento público, la revolución de las tecnologías de la información y la comunicación que promueven el paso del conocimiento universitario al conocimiento pluriuniversitario ${ }^{13}$, el detrimento del proyecto nacional y con ello el debilitamiento de la universidad pública, la descentralización del poder de los docentes que conlleva a la priorización de los administradores encargados de convertir la universidad en una empresa y una profunda ruptura en la identidad de la universidad.

Al respecto conviene agregar que la calidad como concepto importado de la gestión empresarial se mide en las prácticas de evaluación como un producto o, en mejores términos,

13 Boaventura De Sousa Santos define el conocimiento universitario como aquel caracterizado principalmente por marcos conceptuales disciplinares, con un ethos en el cual es el investigador quien define qué es lo que se debe investigar y como se debe investigar, con la problemática de que su construcción se realiza al margen de las necesidades sociales; por otro lado, De Sousa define el conocimiento pluriuniversitario cuya valides radica en la aplicabilidad que pueda tener en la sociedad, como aquel de naturaleza transdisciplinar y heterogénea. En resumidas cuentas para De Sousa, el paso del conocimiento universitario al conocimiento pluriuniversitario no es más que el cambio de las relaciones entre universidad y sociedad (De Sousa, 2005). como una mercancía cualquiera condicionada por la demanda. La influencia del Sistema de Gestión ISO 9000 así lo confirman, el deseo de organizar las universidades públicas como si fueran empresas, optimizando factores de producción físicos y humanos por medio de la reducción de costes señalan que las nociones implícitas en estos Sistemas de Evaluación no están articuladas precisamente a la concepción de educación como derecho.

Además, ha de considerarse el paso de un enfoque político que fomentó la oferta de ciencia y tecnología, a uno en donde la participación de las empresas en el desarrollo investigativo, científico y tecnológico en lo que ha sido considerado como innovación, empieza a ser la esencia de las comunidades académicas para la generación de lo que se conoce en el léxico empresarial como recursos humanos. En este orden de ideas, lo que se busca a partir de este cambio sustancial es la configuración de un entramado social que permita dar validez a la liberalización de la inversión extranjera y a la privatización del sector público (Casas, 2004).

De otro lado, el afianzamiento del sector productivo y el papel que se le asigna a la universidad introduce en la academia elementos de corte empresarial, garantizados por las políticas educativas de los últimos años. Es común ver en todos los programas universitarios la preponderancia de la calidad y la competitividad, a tal punto de inmiscuirse en el discurso universitario (Casas, 2004).

Según Rosalba Casas, se podría hablar del académico empresario como un nuevo actor en la Universidad con un nuevo contrato social. Un promotor de la privatización y capitalización del conocimiento, encargado de internalizar los valores empresariales en las instituciones universitarias a través del concepto de calidad educativa. 


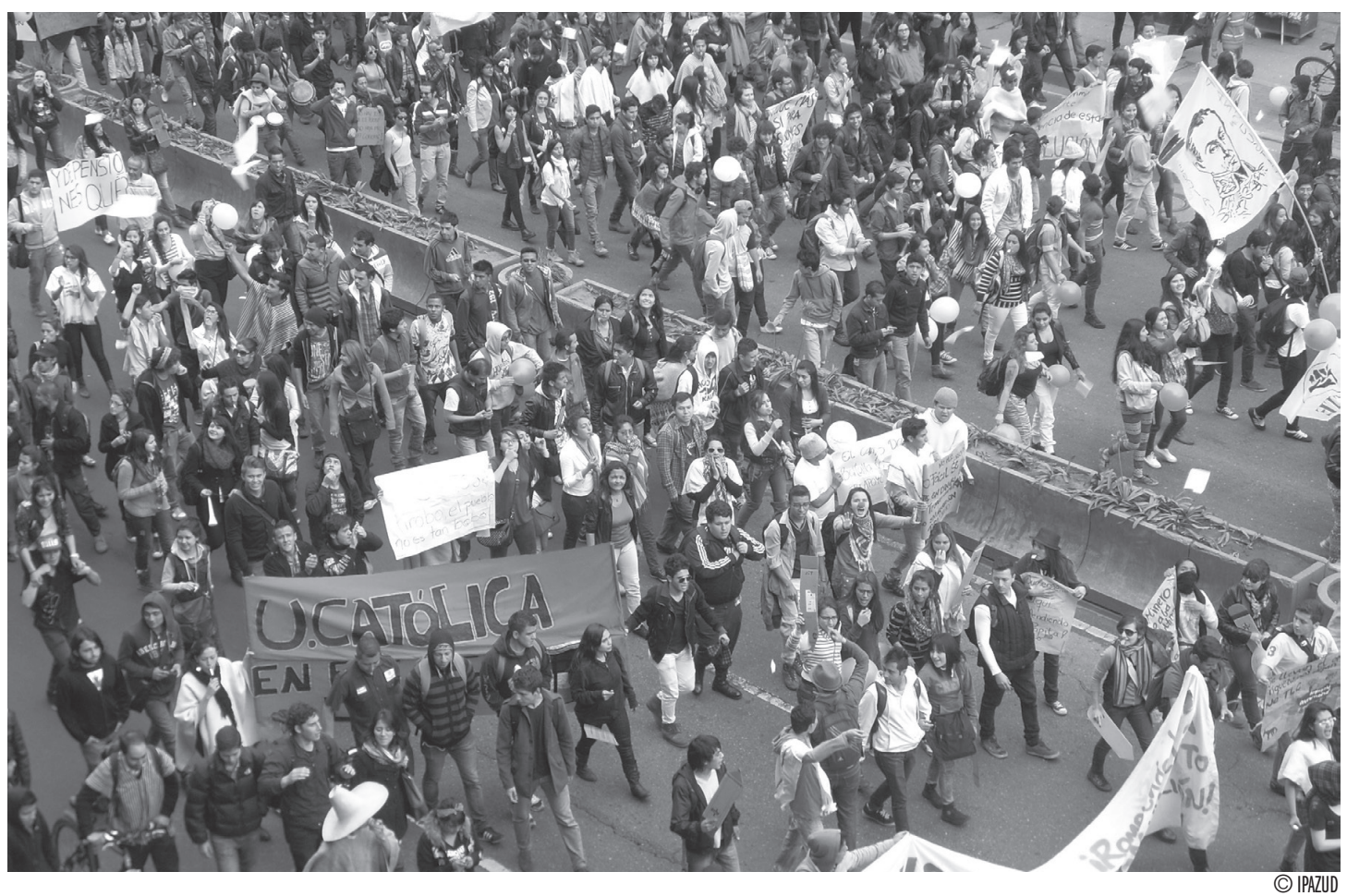

Precisamente por lo mencionado, se podría argumentar que las políticas públicas en este campo reflejan una complejidad conformada por las relaciones que se establecen entre lo público y lo privado, los imaginarios sobre lo público, la intervención de las organizaciones sociales, las fuerzas políticas y el grado de maduración de las relaciones del Estado con los ámbitos educativo y científico ( $\mathrm{He}$ nao, 1999).

Para el caso específico de Colombia, poco distante de lo ocurrido a nivel internacional, ha predominado la debilidad de los vínculos estatales con las necesidades de las comunidades científicas y tecnológicas, aspecto que se evidencia, por un lado, en la poca elaboración de políticas públicas en relación a la educación superior, y por el otro, en la falta de acciones y estrategias que otorguen los recursos necesarios para la superación de los problemas que se muestran en los diagnósticos que se han realizado en torno al tema.
Aquí conviene detenerse un momento a fin de mostrar cómo las políticas públicas en educación superior en Colombia han sido utilizadas elementalmente de las siguientes tres formas: primero, en función de la burocracia, es decir, abarca la organización racional de los medios en función de los fines; en segunda medida, estaría un tipo de organización corporativista en favor del institucionalismo colombiano; y por último, lo que ha sido denominado como Management Public, que consiste en la inserción de las dinámicas propias del sector privado en la gestión pública (Arroyave, 2011).

Con todo y lo anterior, la manera como han sido adoptadas las políticas públicas educativas en Colombia han fomentado una realidad afianzada en problemas tales como el clientelismo, la corrupción y la ilegalidad que determinan el sistema político colombiano y que han debilitado las instituciones en todos los aspectos, por lo que: 
(...) El tercer desafío que enfrentan las políticas públicas en educación superior se da por estas relaciones entre funcionarios, políticos y empresarios. Donde los funcionarios necesitan de los políticos para alcanzar sus cargos y mantenerlos, los políticos necesitan de los empresarios para poder financiar sus campañas y los empresarios de los políticos para crear leyes que beneficien a sus empresas. Estas dinámicas recíprocas han sido uno de los principales obstáculos de las políticas públicas, pues crean un triángulo de hierro que concentra el poder y lo utiliza para el beneficio propio de sus actores, excluyendo a la sociedad civil organizada y no organizada, e impidiendo la construcción de una gobernanza. (Arroyave, 2011, p.102)

En consecuencia, los cambios efectuados a nivel nacional están direccionados por los estándares que impone la división internacional del trabajo en un marco de dominación integral. La educación, entre otros factores sociales relevantes, está siendo homogenizada en aras de un mercado global, por medio de procesos tecnocráticos y burocráticos que por supuesto dan origen a muchas contradicciones sociales económicas y culturales al interior de las naciones.

En suma, el diseño y ejecución de las políticas públicas, de manera ajena a las comunidades académicas, ha propiciado el divorcio entre universidad y sociedad, y la pérdida del sentido crítico y reflexivo que caracterizaba a la universidad pública.

Conceptos como el de calidad de la educación no pueden ser reducidos a estadísticas, estándares cuantitativos o sistemas de medición, estaríamos hablando de una educación instrumental; por consiguiente se debe poner en discusión y en dialogo los diferentes educación universitaria y que no pueden simplificarse a una única verdad en favor de la racionalidad económica.

\section{Reflexiones finales}

A través del análisis de las políticas públicas de educación superior, específicamente de los factores exigidos en las evaluaciones a las que son sometidas las universidades públicas, se vislumbra el propósito de vincular estos establecimientos educativos con las necesidades de los sectores productivos. Esto se logra a partir de dos estrategias recurrentes en las políticas económicas dominantes a nivel mundial: la adaptación de los perfiles de formación a las necesidades del mercado laboral y la articulación entre la investigación desarrollada en las universidades públicas con los requerimientos de los sectores productivos. Estos cambios se encuentran dirigidos hacia la consecución de una mayor inversión en recursos humanos, nuevas estructuras organizativas y de gestión para la producción de bienes y servicios.

Partiendo de estos planteamientos, al interior de las universidades públicas se vienen instalando una serie de reformas profundas que introducen cambios en los currículos, en la orientación de las carreras con un pronunciado favorecimiento de las ingenierías y tecnologías, además de los cambios drásticos en las formas de financiación.

La mercantilización de estas instituciones por medio de la adopción de variadas formas de financiación, sumada a la instalación de esquemas plagados de gestión, conllevan a la priorización de los administradores, quienes han dirigido sus esfuerzos hacia la conversión de la universidad pública en una empresa. De acuerdo con las anteriores problemáticas, se hace necesario repensar la noción de co- 
nocimiento que se ha querido imponer y la finalidad de la universidad frente al ámbito social y político actual.

La pérdida del sentido crítico y reflexivo que caracterizaba la universidad pública le otorga la obligación a la comunidad académica de construir paradigmas y teorías que se contrapongan a los significantes que se han generalizado con el paradigma neoliberal.

Partiendo de lo anunciado, resulta inexorable poner en discusión al interior de las universidades públicas, y entre ellas, los arsenales conceptuales teóricos y metodológicos que giran en torno a la educación terciaria y que no pueden reducirse a la racionalidad económica instrumental que tanto ha pauperizado estos establecimientos educativos.

Para este propósito es necesario que las universidades públicas contrasten la categoría de calidad educativa con el sistema de concepciones impuesto por medio de mecanismos de evaluación, marcos interpretativos coherentes con sus necesidades específicas, de tal manera que las reformas generadas sean el resultado de las necesidades internas de éstas y no el fruto de las imposiciones de los proyectos de las agencias internacionales que se han implantado a nivel mundial.

Al respecto conviene decir que la construcción de marcos interpretativos posibilita la generación de reflexiones y salidas a las engorrosas contradicciones que subsumen la educación superior a las necesidades del capital.

De acuerdo con esto, la denominada innovación organizativa está centrada en los siguientes factores: la efectividad, entendida como la habilidad para producir graduados con competencias relevantes para el mercado laboral; la responsividad, entendida como la habilidad para adaptarse a los cambios; y la eficacia, que supone la obtención de mejores resultados con menores insumos. Estas se erigen como los principales factores promovidos por las instancias certificadoras y las sucesivas políticas de evaluación en respuesta a los dictámenes de las políticas internacionales. Las mismas han incapacitado al Estado en los asuntos económicos, y con ello han favorecido la injerencia de la iniciativa privada ante la abrupta explosión de IES en los noventa.

Conforme a lo planteado, los retos que asume la universidad pública actualmente no son nada más y nada menos que la construcción de salidas y sentidos (ante las encrucijadas derivadas del modelo educativo neoliberal) que permitan reivindicar la importancia de la educación como derecho.

En efecto, para que la evaluación realmente sea un mecanismo efectivo de mejoramiento de las universidades públicas, se deben cambiar los esquemas presupuestales adoptados bajo el auspicio del BM, y considerar las necesidades específicas de este sector. No se puede hablar de mejoramiento cuando estas prácticas no han sido acompañadas por una inversión veraz de recursos, que permita a las universidades públicas proyectarse de acuerdo a sus dinámicas y funcionar en igualdad de condiciones ante la competencia demarcada por las universidades privadas.

\section{Referencias bibliográficas}

- Arroyave, S. (2011). Las políticas públicas en Colombia. Insuficiencias y desafíos. FORUM, (1), 95-111.

- Banco Mundial. (1995). La enseñanza superior. Las lecciones derivadas de la experiencia. Washington, D.C: Banco Mundial. Recuperado de http://bit.ly/1nOMLQR

- Barr, N. (2005). Financiar la Educación Superior. Finanzas \& Desarrollo, 34-37. Recuperado de http://bit.ly/1rxrODk

- Boltanski, L. \& Chiapello, E. (2002). El Nuevo Espíritu del Capitalismo. Madrid, España: Ediciones Akal. 
- Casas, R. (2004). Nuevas orientaciones de las políticas de las universidades en su relación con las empresas. En Mungaray, A., \& Valenti, G. (Coord.), Políticas Públicas y Educación superior (pp.235 - 254). México: Asociación Nacional de Universidades e Instituciones de Educación Superior

- De Moura, C. \& Levy, D. (1997). Higher Education in Latin America and the Caribbean. A strategy paper. Recuperado de http://bit.ly/X698G5

- De Sousa, B. (2005). La universidad en el siglo XXI. Para una reforma democrática y emancipadora de la universidad. México: Universidad Nacional Autónoma de México.

- Del Basto, L. (2004). Una reflexión en torno a la universidad y su acreditación. Revista Electrónica de la Red de Investigación Educativa, 1(1). Recuperado de http://revista.iered.org.

- Didriksson, A. (2000). El valor - conocimiento y la educación superior como empresa económica. En La Universidad del Futuro. Relaciones entre la Educación Superior, la Ciencia y la Tecnología. México: Plaza y Valdés Editores.

- Guido, E. (2005). Acreditación: ¿Calidad o Instrumento de Legitimación para la Educación Superior? Revista Electrónica Actualidades Investigativas en Educación, 5, 1-20. Recuperado de http://bit.ly/11OXmWi

- Harvey, D. (2007). Breve Historia del Neoliberalismo. Recuperado de http://bit.ly/1gLPV3b

- Henao, M. (1999). Políticas públicas y universidad. Estudio sobre las políticas para la capacidad científica de la educación superior en Colombia. Bogotá: Universidad Nacional de Colombia.

- López, F. (2002). Educación superior latinoamericana y organismos internacionales. Un análisis crítico. En El impacto de la globalización y de las políticas educativas en los sistemas de educación superior en América Latina y el Caribe. Cali, Colombia: Universidad de San Buenaventura

- Maldonado, A. (2005). El Banco Mundial y la Educación Superior en los Países en Desarrollo. ¿Cuáles son los peligros y las promesas? Un Análisis del Documento "Peligro y Promesa". Cali, Colombia: Universidad de San Buenaventura.

- Mendoza, J. (1997). Evaluación, acreditación, certificación: instituciones y mecanismos de operación. En Mungaray, A \& Valenti, G (Coord.), Políticas Públicas y Educación Superior (pp.58-75). México: ANUIES. Recuperado de http://bit.ly/1tneMRu

- Ortiz D. (2014). Politicas de Acreditación en el Marco de la Universidad Distrital Francisco José de Caldas. Un Análisis Crítico (2007-2012) (Tesis de pregrado). Universidad Distrital Francisco José de Caldas, Bogotá, Colombia.

- Sanz, F. (2006). La mercantilización de la educación como escenario mundial del espacio europeo de educación superior. Revista Educación, XX1(9), 57-76.

- Sistema Nacional de Acreditación. (2005). Indicadores específicos para los procesos de autoevaluación con fines de acreditación de los programas de educación superior técnicos y tecnológicos. Recuperado de http://bit.ly/1xuo5Na

- Tünnermann, C. \& De Souza, M. (2003). Desafíos de la universidad en la sociedad del conocimiento, cinco años después de la conferencia mundial sobre educación superior. Paper Series, (4). París: Comité Científico Regional para América Latina y el Caribe del Foro de la UNESCO. Recuperado de http://bit.ly/1kl2Ocq

- Zamudio, J. (2008). Políticas Públicas y educación superior. Revista colombiana de educación superior, 1 (0), 79 - 99. Recuperado de http://bit. ly/1zqS5MX 